\title{
Remember the Vessels! Craniofacial Fracture Predicts Risk for Blunt Cerebrovascular Injury
}

\author{
Elina Varjonen, $M D,{ }^{*}$ Frank Bensch, $M D, P b D, \dagger$ Tuomo Pybältö, MD, PhD, $\neq$ \\ Mika Koivikko, MD, PhD, $\S$ and Johanna Snäll, MD, DDS, $P b D / /$
}

Purpose: The risk factors for blunt cerebrovascular injuries (BCVIs) are currently under intensive research, yet it is still controversial who should be screened. This study aimed to determine whether craniofacial fractures are associated with BCVI.

Patients and Methods: This retrospective cohort study focused on patients with suspected polytrauma after whole-body computed tomographic angiography of the cervical arteries. Patients were reviewed for BCVI and craniofacial fractures. Exclusion criteria were hanging injury, gunshot injury or other penetrating injury to the neck, and a cervical fracture on any level. The outcome variable was BCVI, and the main predictor variable was a craniofacial fracture. A secondary predictor variable was a type of craniofacial fracture classified as a facial fracture, skull fracture, or a combination of facial and skull fracture. Other predictor variables were gender, age, and mechanism of injury. In addition, specific craniofacial fractures were analyzed in more detail. The relevance of associations between BCVI and the predictors underwent $\chi^{2}$ testing. Significance was set at .01 .

Results: Four hundred twenty-eight patients 13 to 90 years old during a 12-month period were included in the analysis. Craniofacial fractures occurred in 75 (17.5\%). BCVI occurred significantly more frequently in those with than in those without a craniofacial fracture $(18.6$ vs $7.4 \% ; P=.002)$. Patients with craniofacial fracture had a 4 -fold increased risk for BCVI, whereas those 31 to 50 years old had 3.4-fold increased risk. Type of craniofacial fracture, gender, and mechanism of injury were not associated with BCVI.

Conclusion: Craniofacial fractures are a serious risk factor for BCVI. This research suggests that in patients with any craniofacial fracture and suspected polytrauma, rigorous imaging of cervical arteries in search of BCVI is essential.

(C) 2018 Published by Elsevier Inc. on behalf of the American Association of Oral and Maxillofacial Surgeons

J Oral Maxillofac Surg ם:1.e1-1.e9, 2018

Blunt cerebrovascular injury (BCVI) can lead to devastating neurologic sequelae. BCVI occurs in 1 to $2 \%$ of all patients with blunt trauma, and in severely injured patients, the incidence can be at least twice as high. ${ }^{1-5}$ Early recognition and prompt initiation of treatment of these injuries have resulted in a marked decrease in
*Consultant, Department of Radiology, HUS Medical Imaging Center, University of Helsinki and Helsinki University Hospital, Helsinki, Finland.

†Consultant, Department of Radiology, HUS Medical Imaging Center, University of Helsinki and Helsinki University Hospital, Helsinki, Finland.

$\Varangle$ Consultant, Department of Orthopedics and Traumatology, Töölö Hospital, Helsinki University Hospital, Helsinki, Finland.

$\S$ Radiologist-in-Chief and Adjunct Professor, Department of Radiology, HUS Medical Imaging Center, University of Helsinki and Helsinki University Hospital, Helsinki, Finland.

\|Consultant, Department of Oral and Maxillofacial Diseases, University of Helsinki and Helsinki University Hospital, Helsinki, Finland.
Conflict of Interest Disclosures: None of the authors have any relevant financial relationship(s) with a commercial interest.

Address correspondence and reprint requests to Dr Varjonen: Department of Radiology, Töölö Trauma Center, Topeliuksenkatu 5, 00029 HUS, Finland; e-mail: elina.a.varjonen@hus.fi

Received January 12018

Accepted March 232018

(c) 2018 Published by Elsevier Inc. on behalf of the American Association of Oral and Maxillofacial Surgeons

0278-2391/18/30293-3

hitps://doi.org/10.1016/i.joms.2018.03.035 

common BCVI-associated craniomaxillofacial pattern. ${ }^{17}$

A correlation between skull base fractures and BCVI has been repeatedly reported in previous studies. $^{1,14,15,18,19}$ These studies were consistent in indicating skull base fractures as a risk factor for BCVI, although fracture types varied. Previously, the focus was on petrous bone fractures and carotid canal involvement, ${ }^{18-20}$ whereas more recent investigations have taken into account other parts of the skull base $^{13-15}$ and fronto-orbital area. ${ }^{11}$ York et al $^{21}$ reported BCVI rates for all types of skull fractures.

In bony craniofacial trauma, fracture lines often continue across facial and cranial bones, so the craniofacial region can be considered a single unit. Therefore, in contrast to previous studies, the present analyses included any type of facial and skull fracture, with the aim of analyzing possible correlations between BCVI and craniofacial fractures and evaluating BCVI incidence in different types of craniofacial fractures. The hypothesis was that there would be an association between craniofacial fractures and BCVI. Specific aims for the study were to analyze whether some facial fracture subtypes correlate with an increased risk for BCVI.

\section{Patients and Methods}

\section{STUDY DESIGN}

For this retrospective study, the cohort consisted of patients with suspected blunt high-energy polytrauma who were admitted to a level 1 trauma center (Töölö Trauma Center, Helsinki University Hospital, Helsinki, Finland) in accord with a trauma alarm protocol from May 2015 through May 2016. These included patients with a mechanism of injury serious enough to require computed tomography (CT) of the whole body. All patients were subjected to split-bolus whole-body 64-slice CT (Discovery CT 750 HD, GE Healthcare, Milwaukee, WI) including angiography (aWBCT), which includes a continuous scan from the skull base to the ischium in simultaneous arterial and portal venous phases. Table 1 presents the imaging protocol. Data for patients imaged by aWBCT, in addition to their demographic data, clinical findings, and initial reports, were retrospectively retrieved from the Picture Archiving and Communications System (Impax 6, Agfa HealthCare NV, Mortsel, Belgium) and electronic patient files.

Exclusion criteria were hanging injury, gunshot injury or other penetrating injury to the neck region, and cervical fracture at any level.

All CT studies were reviewed by 2 board-certified radiologists with 12 and 6 years of experience in trauma radiology, respectively, who were blinded to the initial reports. Any discrepancies were resolved by consensus.

\section{STUDY VARIABLES}

The main outcome variable was BCVI. The main predictor variable was a craniofacial fracture. A secondary predictor variable was type of craniofacial fracture classified as a facial fracture, skull fracture, or a combination of facial and skull fractures. Other predictor variables were gender, age, mechanism of injury, and craniofacial fracture subgroups.

Patients with craniofacial fractures were identified and their fractures were divided into 4 facial fracture subgroups and 3 skull fracture subgroups. Facial fractures consisted of 1) combined facial fractures 


\section{Table 1. IMAGING PROTOCOL OF BODY COMPUTED TOMOGRAPHIC ANGIOGRAPHY OF CERVICAL \\ ARTERIES}

Split-bolus imaging protocol

Scan area

Circle of Willis and ischium (lower extremities on request)

Contrast enhancement

Cervical spine in arterial phase

Body in simultaneous arterial and venous phases $80 \mathrm{~mL}+50-\mathrm{mL}$ contrast medium bolus (Omnipaque $350 \mathrm{mg} / \mathrm{mL}+$ iohexol) at 22-second interval

Contrast delay

Cervical spine, SmartPrep* with 130-HU threshold

Body, 45-second fixed delay

Image acquisition parameters

Slice thickness, $0.625 \mathrm{~mm}$

Range, 120-700 mA

Pitch, $39.37 \mathrm{~mm} /$ rotation

Table feed: neck, $98.4 \mathrm{~mm} / \mathrm{second}$; body, $137.5 \mathrm{~mm} / \mathrm{second}$

Neck: kV 100, NI 40

Body: kV 120, NI 50

Reformatted series

Axial, coronal, sagittal planes; vascular and bone windows

Reformatted slice thickness

Cervical spine: axial, $1.25 \mathrm{~mm}$; coronal and sagittal, $1.5 \mathrm{~mm}$

Cervical vessels, $2 \mathrm{~mm}$ coronal and sagittal

Body, $3 \mathrm{~mm}$ coronal and sagittal

* GE Healthcare Ireland (Cork, Ireland).

Varjonen et al. Blunt Cerebrovascular Injury. J Oral Maxillofac Surg 2018.

(mandibular and midfacial fractures, midfacial and upper third fractures, and panfacial fractures extending to all facial thirds); 2) upper third fractures (fractures of the frontal sinus, orbital roof, or anterior skull base); 3) midfacial fractures (multiple midfacial fractures, Le Fort I to III, naso-orbito-ethmoidal, zygomatic, and orbital fractures other than the roof or nasal fractures); or 4) lower facial fractures (exclusively mandibular fractures). Skull fractures consisted of 1) basilar skull fractures (fractures of the sphenoid, petrous temporal bone, or basilar portion of the occipital bone); 2) other skull fractures; or 3) complex skull fractures (combination of basilar skull fractures and other skull fractures). Specific facial fractures and fractures involving the carotid canal and foramen magnum were documented separately.

\section{STATISTICAL ANALYSIS}

For statistical analysis, SPSS 24.0 (IBM Corp, Armonk, NY) was used. The relevance of associations between BCVI and craniofacial fractures, facial and skull fracture subtypes, gender, age, and mechanism of trauma was tested with the $\chi^{2}$ test. Significance level was set at .01. Multivariate logistic regression analysis was performed to test the relation between BCVI and the explanatory variables gender, age, mechanism of injury, and any craniofacial fracture.

\section{ETHICAL APPROVAL}

The internal review board of the Division of Musculoskeletal Surgery at the Helsinki University Hospital approved the study. Patient-informed consent was waived, because the study was retrospective. The study followed the principles of the Declaration of Helsinki.

\section{Results}

Of the 465 patients, 7 were excluded for poor image quality or lack of intravenous contrast media and 30 were excluded for cervical spine fractures. None of the patients had sustained a hanging, gunshot, or other penetrating injury to the neck region. Thus, 428 patients were accepted for the final analyses.

Table 2 presents descriptive statistics for the 428 patients. Most patients (71\%) were male, and mean

\section{Table 2. DESCRIPTIVE STATISTICS FOR 428 PATIENTS WITH TRAUMA}

\begin{tabular}{lrr} 
& $\mathrm{n}$ & $\%$ \\
\hline Gender & & \\
\hline Male & 304 & 71.0 \\
\hline Female & 124 & 29.0 \\
\hline Age (yr) & & \\
$\quad$ Mean & 41.9 & \\
Range & $13-90$ & \\
\hline Age groups (yr) & & \\
13-30 & 133 & 31.1 \\
\hline $31-50$ & 156 & 36.4 \\
\hline $51-70$ & 111 & 25.9 \\
\hline $71-90$ & 28 & 6.5 \\
\hline Mechanism of trauma & & \\
Motor vehicle accident & 199 & 46.5 \\
\hline Fall from height & 86 & 20.1 \\
\hline Bicycle accident & 35 & 8.2 \\
\hline Pedestrian traffic injuries & 24 & 5.6 \\
\hline Fall on stairs & 19 & 4.4 \\
\hline Assault & 14 & 3.3 \\
\hline Other & 51 & 11.9 \\
\hline Craniofacial fracture & 75 & 17.5 \\
\hline Facial fracture & 42 & 9.8 \\
\hline Skull fracture & 19 & 4.4 \\
\hline Facial + skull fracture & 14 & 3.4 \\
\hline BCVI & 40 & 9.3 \\
\hline Abbriation BCVI, & & \\
\hline
\end{tabular}

Abbreviation: BCVI, blunt cerebrovascular injury.

Varjonen et al. Blunt Cerebrovascular Injury. J Oral Maxillofac Surg 2018. 
age was 41.9 years. The most common mechanism of injury was motor vehicle accident (46.5\%), followed by fall from a height (20.1\%) and bicycle accident (8.2\%). Other injury mechanisms were pedestrian traffic injuries (5.6\%), a fall on the stairs (4.4\%), and assault (3.3\%). The remaining $11.9 \%$ fell under other injury mechanisms, in which the mechanism was unknown or the trauma was considered of relatively low energy. Such mechanisms include falls from low heights or on even on the ground, sports injuries, and being struck by or crushed between blunt objects. Craniofacial fractures had occurred in 75 patients (17.5\%), of whom $42(58.3 \%)$ had a facial fracture, 19 (25.3\%) had a skull fracture, and 14 (18.7\%) had facial and skull fractures, and 40 (9.3\%) had BCVI.

Table 3 presents the association between BCVI and its predictors. BCVI was evident in $18.6 \%$ of patients with craniofacial fracture, reaching significance $(P=.002)$. BCVI occurred most frequently with skull fractures (21.1\%), followed by facial fractures (19.0\%) and combinations of facial and skull fractures (14.3\%), although the differences among these 3 patient groups were nonsignificant $(P=.882)$. No meaningful associations emerged between BCVI and gender or mechanism of trauma. Table 4 presents a logistic regression analysis between BCVI and gender, age group, mechanism of injury, and craniofacial fractures. Analysis showed a 4-fold higher risk of BCVI in patients with craniofacial fracture than in those with other trauma (odds ratio $[\mathrm{OR}]=4.096$; $95 \%$ confidence interval $[\mathrm{CI}], 1.866-8.993 ; P<.001)$. Patients 31 to 50 years old had a 3.4 -fold higher risk than their reference group (13 to $30 \mathrm{yr}$; OR $=3.426 ; 95 \% \mathrm{CI}$, $1.356-8.656 ; P=.009)$.

Table 5 presents associations between craniofacial fracture subgroups and BCVI. In the 56 patients with facial fractures, BCVI most commonly occurred in combined facial (20.0\%) and midfacial (20.0\%) fractures. None with exclusively upper or lower facial fractures showed BCVI. No statistically relevant differences appeared between BCVI and facial fracture subgroups. Of the 33 patients with skull fractures, BCVI occurred most frequently in those with complex skull fractures (37.5\%), followed by basilar skull fractures (14.3\%), but in no other types of skull fracture. The correlation between BCVI and complex skull fractures was almost significant $(P=.033)$.

Table 6 presents specific facial fractures and fractures extending into the carotid canal and foramen magnum. Further analysis showed that all BCVIs in facial fractures occurred in isolated zygomaticoorbital fractures $(35.7 \%)$, combined facial fractures

\section{Table 3. ASSOCIATION BETWEEN BCVI AND PREDICTORS IN 428 PATIENTS WITH TRAUMA}

Q5

\begin{tabular}{|c|c|c|c|c|c|}
\hline & $\mathrm{n}$ & BCVI Present & $\%$ & BCVI in 40 Patients, \% & $P$ Value \\
\hline & & 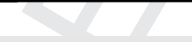 & & & \\
\hline Gender & & & & & .110 \\
\hline Male & 304 & 24 & 7.9 & 60.0 & \\
\hline Female & 124 & 16 & 12.9 & 40.0 & \\
\hline Age groups (yr) & & $\nabla$ & & & .110 \\
\hline $13-30$ & 133 & 7 & 5.3 & 17.5 & \\
\hline $31-50$ & 156 & 21 & 13.5 & 52.5 & \\
\hline $51-70$ & 111 & 9 & 8.1 & 22.5 & \\
\hline $71-90$ & 28 & 3 & 10.7 & 7.5 & \\
\hline Mechanism of trauma & & & & & .500 \\
\hline Motor vehicle accident & 199 & 23 & 11.6 & 57.5 & \\
\hline Fall from height & 86 & 9 & 10.5 & 22.5 & \\
\hline Bicycle accident & 35 & 1 & 2.9 & 2.5 & \\
\hline Pedestrian traffic injuries & 24 & 1 & 4.2 & 2.5 & \\
\hline Fall on stairs & 19 & 2 & 10.5 & 5 & \\
\hline Assault & 14 & 0 & 0 & 0 & \\
\hline Other & 51 & 4 & 7.8 & 10 & \\
\hline \multicolumn{6}{|l|}{ Craniofacial fracture } \\
\hline Yes & 75 & 14 & 18.6 & 35.0 & .002 \\
\hline No & 353 & 26 & 7.4 & 65.0 & \\
\hline Facial fracture & 42 & 8 & 19.0 & 20 & .882 \\
\hline Skull fracture & 19 & 4 & 21.1 & 10 & \\
\hline Facial + skull fracture & 14 & 2 & 14.3 & 5 & \\
\hline
\end{tabular}

Note: Significance level was set at .01.

Abbreviation: BCVI, blunt cerebrovascular injury.

Varjonen et al. Blunt Cerebrovascular Injury. J Oral Maxillofac Surg 2018. 

Table 4. MULTIVARIATE REGRESSION ANALYSIS FOR
BLUNT CEREBROVASCULAR INJURIES IN 428 PATIENTS WITH TRAUMA

\begin{tabular}{|c|c|c|c|c|}
\hline & \multirow[b]{2}{*}{ OR } & \multicolumn{2}{|c|}{$95 \% \mathrm{CI}$ for OR } & \multirow[b]{2}{*}{$P$ Value } \\
\hline & & Lower & Upper & \\
\hline Gender (ref, female) & 0.460 & 0.223 & 0.946 & .035 \\
\hline \multicolumn{5}{|l|}{$\begin{array}{l}\text { Age groups } \\
\text { (ref, } 13-30 \mathrm{yr})\end{array}$} \\
\hline $31-50 \mathrm{yr}$ & 3.426 & 1.356 & 8.656 & .009 \\
\hline $51-70 \mathrm{yr}$ & 2.179 & 0.746 & 6.370 & .155 \\
\hline $71-90 \mathrm{yr}$ & 2.432 & 0.531 & 11.145 & .253 \\
\hline \multicolumn{5}{|l|}{$\begin{array}{l}\text { Mechanism of injury } \\
\text { (ref, MVA) }\end{array}$} \\
\hline Fall from height & 0.701 & 0.293 & 1.675 & .424 \\
\hline Bicycle accident & 0.110 & 0.013 & 0.908 & .040 \\
\hline $\begin{array}{l}\text { Pedestrian traffic } \\
\text { injuries }\end{array}$ & 0.170 & 0.020 & 1.449 & .105 \\
\hline Fall on stairs & 0.491 & 0.092 & 2.622 & .405 \\
\hline Assault & 0.000 & 0.000 & & .998 \\
\hline Other & 0.452 & 0.143 & 1.433 & .178 \\
\hline $\begin{array}{l}\text { Craniofacial fracture } \\
\text { (ref, yes) }\end{array}$ & 4.096 & 1.866 & 8.993 & $<.001$ \\
\hline
\end{tabular}

Note: Significance level was set at .01.

Abbreviations: CI, confidence interval; MVA, motor vehicle accident; OR, odds ratio; ref, reference.

Varjonen et al. Blunt Cerebrovascular Injury. J Oral Maxillofac Surg 2018.

(20.0\%), and isolated orbital fractures (12.5\%). The number of specific facial fractures was insufficient for statistical analysis. Only 1 patient with a skull base fracture extending to the foramen magnum
(12.5\%) had BCVI, and none of the 5 patients with a fracture extending to the carotid canal had BCVI.

\section{Discussion}

The purpose of this study was to analyze possible correlations between BCVI and craniofacial fractures and to evaluate the incidence of BCVI in different types of craniofacial fractures, with the hypothesis of there being an association between craniofacial fractures and BCVI. Furthermore, the authors investigated a possible correlation of facial fracture subtypes to BCVI.

The present study of craniofacial fractures and BCVI showed a strong correlation, with BCVI occurring 2.5 times more frequently in patients with craniofacial fracture than in those with all other types of severe trauma. Nearly 1 in 5 patients with craniofacial fracture (18.6\%) was diagnosed with BCVI. There was no difference between facial fractures and skull fractures with regard to BCVI. Logistic regression showed a 4-fold higher risk of BCVI for craniofacial fractures compared with all other trauma.

Thus, the present results are in line with those of Buch et $\mathrm{al}^{14}$ who stated that BCVI can occur in up to $11 \%$ of patients with blunt trauma injuries. However, overall BCVI incidence was considerably higher (9.3\%) in the present study than in previous studies. $^{1-5}$ One possible explanation for the higher incidence of BCVI could be the authors' institution serving as a level 1 trauma center and a tertiary hospital and therefore having a higher incidence of severe trauma. Furthermore, the authors' guidelines

\section{Table 5. ASSOCIATION BETWEEN BCVI AND SUBGROUPS OF CRANIOFACIAL FRACTURES IN 75 PATIENTS WITH} CRANIOFACIAL FRACTURE

\begin{tabular}{|c|c|c|c|c|c|}
\hline & $\mathrm{n}$ & BCVI Present & $\%$ & BCVI in 14 Patients, \% & $P$ Value \\
\hline \multicolumn{6}{|l|}{ Facial fracture } \\
\hline Any $(n=56)$ & 56 & 10 & 17.9 & 71.4 & .757 \\
\hline Combined facial & 20 & 4 & 20.0 & 28.6 & .090 \\
\hline Upper & 1 & 0 & & & \\
\hline Midfacial & 30 & 6 & 20.0 & 42.9 & \\
\hline Lower & 5 & 0 & & & \\
\hline \multicolumn{6}{|l|}{ Skull fracture } \\
\hline Any $(\mathrm{n}=33)$ & 33 & 6 & 18.2 & 42.9 & .924 \\
\hline Basilar skull fracture* & 21 & 3 & 14.3 & 21.4 & .033 \\
\hline Other & 4 & 0 & & & \\
\hline Complex skull fracture & 8 & 3 & 37.5 & 21.4 & \\
\hline
\end{tabular}

Note: Significance level was set at .01.

Abbreviation: BCVI, blunt cerebrovascular injury.

* Fracture of the sphenoid, petrous, temporal, clivus, or occipital condyle.

$\dagger$ Basilar skull fracture plus other skull fracture.

Varjonen et al. Blunt Cerebrovascular Injury. J Oral Maxillofac Surg 2018. 
Table 6. SPECIFIC FACIAL FRACTURES AND FRACTURES EXTENDING TO THE CAROTID CANAL AND FORAMEN MAGNUM AND BCVI IN 75 PATIENTS

\begin{tabular}{|c|c|c|c|c|}
\hline & $\mathrm{n}$ & $\begin{array}{c}\text { BCVI } \\
\text { Present }\end{array}$ & $\%$ & $\begin{array}{c}P \\
\text { Value }\end{array}$ \\
\hline Facial fractures $(n=56)$ & & & & NA \\
\hline Combined facial & 20 & 4 & 20.0 & \\
\hline Upper third & 1 & 0 & & \\
\hline Multiple midfacial & 4 & 0 & & \\
\hline Exclusively orbital & 8 & 1 & 12.5 & \\
\hline $\begin{array}{l}\text { Exclusively } \\
\text { zygomatico-orbital }\end{array}$ & 14 & 5 & 35.7 & \\
\hline Exclusively maxillary & 1 & 0 & & \\
\hline Exclusively nasal & 3 & 0 & & \\
\hline Exclusively condylar & 2 & 0 & & \\
\hline $\begin{array}{l}\text { Exclusively } \\
\text { non-condylar }\end{array}$ & 3 & 0 & & \\
\hline Skull fractures $(n=33)$ & & & & .084 \\
\hline Carotid canal & 5 & 0 & & \\
\hline Foramen magnum & 8 & 1 & 12.5 & \\
\hline $\begin{array}{l}\text { Fracture not involving } \\
\text { foramen }\end{array}$ & 20 & 5 & 25.0 & \\
\hline
\end{tabular}

Note: Significance level was set at .01.

Abbreviations: BCVI, blunt cerebrovascular injury; NA,

Varjonen et al. Blunt Cerebrovascular Injury. J Oral Maxillofac Surg 2018.

for BCVI screening in patients with high-energy trauma seem liberal; in addition to the Denver criteria, the authors included thoracic and lower cervical spine trauma. In the authors' unit, even if the trauma alarm protocol has not been triggered, the attending trauma surgeon can order an aWBCT when clinical signs or mechanism of trauma indicate possible severe trauma. Thus, the liberal screening protocol could have increased the number of imaged patients and diagnosed BCVIs, especially among facial fracture cases.

Buch et $\mathrm{al}^{14}$ reported that $87 \%$ of their patients with BCVI also had cervical or skull base fractures or a combination of the 2 , but concluded that isolated midface fractures in the absence of cervical spine or skull base fractures were not associated with underlying BCVI. However, the only facial injuries observed in their study were complex facial fractures with midface instability. The authors excluded cervical fractures to focus on the effect of craniofacial fractures alone. Occurrence was almost evenly divided among isolated facial fractures, isolated cranial fractures, and combinations of the 2 groups. Further analysis showed a high occurrence of BCVI in combined facial fractures and midfacial fractures, especially in zygomatico-orbital fractures (Figs 1, 2). Based on these results, facial fractures should be considered an independent risk factor for BCVI. For more accurate conclusions about different types of craniofacial fractures, a larger number of patients with specific fracture types is required.

Recent studies have emphasized the relevance of facial fractures in BCVIs, such as mandibular and especially extracapsular condylar fractures ${ }^{15,22}$ and maxillary fractures involving pterygoid plates. ${ }^{15}$ Kang et $\mathrm{al}^{23}$ described the injury mechanism of the internal carotid artery (ICA) in conjunction with Le Fort I osteotomy. Owing to the anatomic proximity of the ICA to the foramen lacerum and the pterygoid plate, the artery can be directly damaged if a sharp bony edge causes shearing near the skull base. This is in line with the findings of Mundinger et $\mathrm{al}^{15}$ who concluded that in patients with facial fracture, risk for BCVI without applying screening criteria was higher in patients with Le Fort I fractures. The same study reported corresponding findings in patients with mandibular subcondylar fractures. Vranis et $\mathrm{al}^{22}$ studied condyle fractures in more detail and found that direct injury caused by bony fragments, especially displaced extracapsular condyle fractures, increased the risk for BCVI. According to these studies, Le Fort I fractures and extracapsular mandibular condylar fractures, in particular, can lead to localized ICA damage.

The authors found no associations between BCVI and these fracture subtypes. The present study showed a high occurrence of BCVI, especially in zygomatico-orbital fractures, indicating that a facial fracture is a marker of substantial trauma energy, which in turn increases the likelihood of BCVI, even when the fracture does not cause direct mechanical damage to a vessel.

Others have reported an association between skull base fractures and BCVI. ${ }^{1,14,15,18,19}$ In the present study, the authors analyzed all types of cranial fractures. BCVI occurred most frequently in complex skull fractures (combination of basilar skull fractures and other skull fractures; 37.5\%) and basilar skull fractures $(14.2 \%)$, but none of the patients with exclusively other skull fracture types showed BCVI. This differs from the findings of York et $\mathrm{al}^{21}$ who reported a high BCVI rate in fractures in other parts of the skull, with $29 \%$ of patients with non-basilar skull fractures having ICA injury. Carotid canal fractures also have been implicated as a risk factor for BCVI. Petrous bone fractures and carotid canal involvement can lacerate a blood vessel, especially in the intraosteal segment. ${ }^{20}$ York et $\mathrm{al}^{21}$ also evaluated the incidence of ICA injury and skull fractures, establishing that in skull fractures with carotid canal involvement (35\%), injury to the ICA was twice as frequent as without canal involvement (15\%). Interestingly, in the present study, none of the 5 patients with carotid canal involvement and only 1 of 8 patients with a fracture extending to the foramen magnum had BCVI. The number of these patients was small, and further investigation with a 
FIGURE 1. A 25-year-old unconscious woman was admitted with an unclear injury mechanism. Radiologic imaging depicted a left zygomatic arch fracture (broad arrow), right mandibular parasymphyseal fracture (thin arrow), and dental injuries to the upper incisors (arrowhead).

Varjonen et al. Blunt Cerebrovascular Injury. J Oral Maxillofac Surg 2018.

larger cohort of patients might clarify any associations between skull fracture subtypes and BCVI.

The question arises as to whether BCVI correlates with a certain type of injury or requires a certain amount of trauma energy. Even minor craniofacial trauma has coincided with serious carotid injury. One case study described a healthy young man with a non-dislocated mandibular double fracture as a result of a single punch, who was diagnosed with carotid dissection and acute secondary embolic infarcts. ${ }^{24}$ Considering that report and the variation in craniofacial fractures with BCVI in the present and previous studies, the energy required to cause any craniofacial fracture seems sufficient to cause an associated BCVI. Interestingly, a previous study found that a long styloid process of the temporal bone can contribute to the pathogenesis of cervical carotid dissection. $^{25}$ Thus, taking into consideration that hyperextension, rotation, and lateral flexion of the neck predispose to BCVI, more detailed evaluation of a local anatomic association is required. Further investigation of the level of BCVI and possible vectors for trauma energy would probably shed some light on these associations.

Controversy still exists concerning which patients with trauma are at risk for BCVI and for whom screening is thus indicated. Current BCVI screening guidelines allow for BCVI being missed and risk for stroke. Thus, more liberalized screening for BCVI during initial CT in patients with trauma and signs of typical mechanisms or high-energy trauma is warranted. ${ }^{2}$ The overall incidence of BCVI has increased during the past decade, in part because of the increased availability and accuracy of cervical CTA in 
785

786

787

788

789

790

791

792

793

794

795

796

797

798

799

800

801

802

803

804

805

806

807

808

809

810

811

812

813

814

815

816

817

818

819

820

821

822

823

824

825

826

827

828

829

830

831

832

833

834

835

836

837

838

839

840

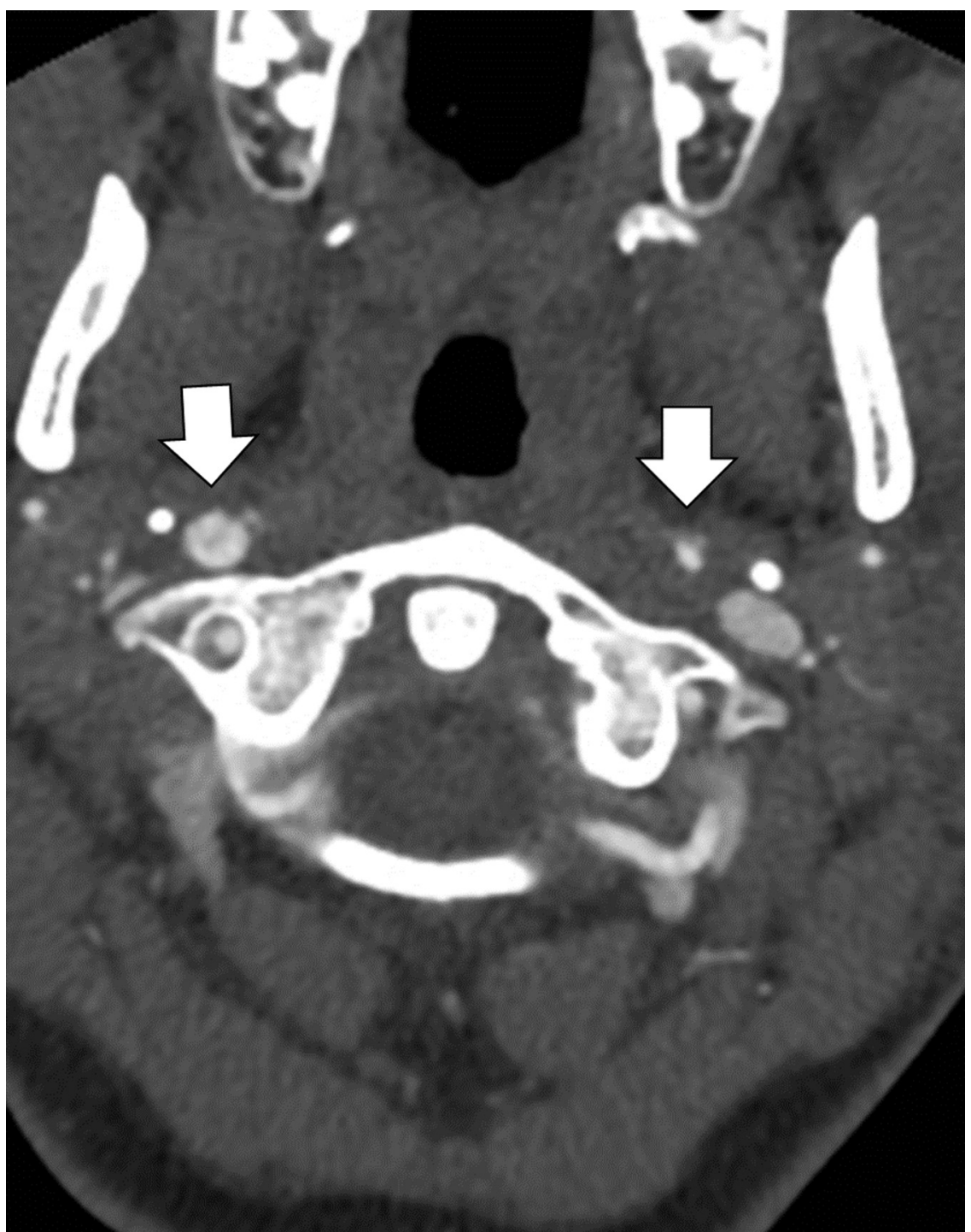

FIGURE 2. Cervical computed tomographic angiography displayed blunt cerebrovascular injury (Biffl grade 2) in the 2 internal carotid arteries (arrows).

Varjonen et al. Blunt Cerebrovascular Injury. J Oral Maxillofac Surg 2018.

combination with an increasing index of suspicion and increased experience among trauma surgeons treating these injuries. ${ }^{1,26}$

Despite greater academic interest in this topic, unambiguous evidence of associations between craniofacial injuries and BCVIs is lacking. The present study found that craniofacial fractures are a notable risk factor for BCVI, without relevant differences in risk between fracture subtypes. Therefore, in all patients with polytrauma and craniofacial fractures, BCVI should be excluded.

\section{References}

1. Berne JD, Cook A, Rowe SA, et al: A multivariate logistic regression analysis of risk factors for blunt cerebrovascular injury. J Vasc Surg 51:57, 2010
2. Bruns BR, Tesoriero R, Kufera J, et al: Blunt cerebrovascular injury screening guidelines: What are we willing to miss? J Trauma Acute Care Surg 76:691, 2014

3. Miller PR, Fabian TC, Croce MA, et al: Prospective screening for blunt cerebrovascular injuries: Analysis of diagnostic modalities and outcomes. Ann Surg 236:386, 2002

4. Biffl WL, Ray CE Jr, Moore EE, et al: Treatment-related outcomes from blunt cerebrovascular injuries: Importance of routine follow-up arteriography. Ann Surg 235:699, 2002

5. Fleck SK, Langner S, Baldauf J, et al: Incidence of blunt craniocertrauma imaging with adapted computed tomography angiography. Neurosurgery 69:615, 2011

6. Stein DM, Boswell S, Sliker CW, et al: Blunt cerebrovascular injuries: Does treatment always matter? J Trauma 66: 132, 2009

7. Miller PR, Fabian TC, Bee TK, et al: Blunt cerebrovascular injuries: Diagnosis and treatment. J Trauma 51:279, 2001

8. Eastman AL, Muraliraj V, Sperry JL, et al: CTA-based screening reduces time to diagnosis and stroke rate in blunt cervical vascular injury. J Trauma 67:551, 2009 vical artery injuries: Use of whole-body computed tomography 
9. Fabian TC, Patton JH Jr, Croce MA, et al: Blunt carotid injury. Importance of early diagnosis and anticoagulant therapy. Ann Surg 223:513, 1996

10. Bromberg WJ, Collier BC, Diebel LN, et al: Blunt cerebrovascular injury practice management guidelines: The Eastern Association for the Surgery of Trauma. J Trauma $68: 471,2010$

11. Burlew CC, Biffl WL, Moore EE, et al: Blunt cerebrovascular injuries: Redefining screening criteria in the era of noninvasive diagnosis. J Trauma Acute Care Surg 72:330, 2012

12. Burlew CC, Biffl WL: Imaging for blunt carotid and vertebral artery injuries. Surg Clin North Am 91:217, 2011

13. Nakajima H, Nemoto M, Torio T, et al: Factors associated with blunt cerebrovascular injury in patients with cervical spine injury. Neurol Med Chir (Tokyo) 54:379, 2014

14. Buch K, Nguyen T, Mahoney E, et al: Association between cervical spine and skull-base fractures and blunt cerebrovascular injury. Eur Radiol 26:524, 2016

15. Mundinger GS, Dorafshar AH, Gilson MM, et al: Blunt-mechanism facial fracture patterns associated with internal carotid artery injuries: Recommendations for additional screening criteria based on analysis of 4,398 patients. J Oral Maxillofac Surg 71: 2092, 2013

16. Geddes AE, Burlew CC, Wagenaar AE, et al: Expanded screening criteria for blunt cerebrovascular injury: A bigger impact than anticipated. Am J Surg 212:1167, 2016

17. Kelts G, Maturo S, Couch ME, et al: Blunt cerebrovascular injury following craniomaxillofacial fractures: A systematic review. Laryngoscope 127:79, 2017
18. Biffl WL, Moore EE, Ryu RK, et al: The unrecognized epidemic of blunt carotid arterial injuries: Early diagnosis improves neurologic outcome. Ann Surg 228:462, 1998

19. Cothren CC, Biffl WL, Moore EE, et al: Treatment for blunt cerebrovascular injuries: Equivalence of anticoagulation and antiplatelet agents. Arch Surg 144:685, 2009

20. Carter DA, Mehelas TJ, Savolaine ER, et al: Basal skull fracture with traumatic polycranial neuropathy and occluded left carotid artery: Significance of fractures along the course of the carotid artery. J Trauma 44:230, 1998

21. York G, Barboriak D, Petrella J, et al: Association of internal carotid artery injury with carotid canal fractures in patients with head trauma. AJR Am J Roentgenol 184:1672, 2005

22. Vranis NM, Mundinger GS, Bellamy JL, et al: Extracapsular mandibular condyle fractures are associated with severe blunt internal carotid artery injury: Analysis of 605 patients. Plast Reconstr Surg 136:811, 2015

23. Kang SY, Lin EM, Marentette LJ: Importance of complete pterygomaxillary separation in the Le Fort I osteotomy: An anatomic report. Skull Base 19:273, 2009

24. Murabit A, Tredget EE: Blunt carotid artery injury after minor facial trauma. Can J Plast Surg 20:194, 2012

25. Raser JM, Mullen MT, Kasner SE, et al: Cervical carotid artery dissection is associated with styloid process length. Neurology 77:2061, 2011

26. Berne JD, Reuland KS, Villarreal DH, et al: Sixteen-slice multidetector computed tomographic angiography improves the accuracy of screening for blunt cerebrovascular injury. J Trauma 60:1204, 2006 